\title{
THE FEBRUARY MEETING IN NEW YORK
}

The two hundred fifty-third regular meeting of the Society was held at Columbia University, on Saturday, February 26, 1927, extending through the usual morning and afternoon sessions. The attendance included the following seventy-two members:

W. L. Ayres, W. M. Bond, Carrie, L. D. Cummings, Dadourian, Dehn, Doermann, Douglas, Eisenhart, Ettlinger, Fiske, Fite, D. A. Flanders, Fort, Gehman, Gill, Glenn, Gronwall, C. C. Grove, Guggenbühl, Haskins, Hedlund, Himwich, Joffe, R. A. Johnson, Kasner, Kellogg, Kholodovsky, Kline, Koopman, Kuhn, Langman, Lefschetz, Littauer, Luby, MacColl, MacNeish, Michael, T. W. Moore, Mullins, F. H. Murray, K. E. O'Brien, Pederson, Pfeiffer, Pierpont, Pooler, Post, R. G. Putnam, Raudenbush, Reddick, Reid, R. G. D. Richardson, D. E. Richmond, Ritt, Seely, Skolnik, Smail, M. H. Stone, Takasu, E. M. Thomas, T. Y. Thomas, Tracey, Vallarta, Veblen, Evelyn Walker, Wedderburn, Weida, Weisner, Whittemore, Wiener, W. A. Wilson, J. W. Young.

There was no meeting of the Council or of the Trustees of the Society.

Professor Wiener presided at the morning session, and Vice-President Kellogg in the afternoon.

At the request of the Program Committee, Professor D. J. Struik, of the Deft Technical School, now visiting professor at the Massachusetts Institute of Technology, delivered an address, at the beginning of the afternoon session, entitled The geometry of linear displacements; this paper will be published in full in an early issue of this Bulletin.

Titles and abstracts of the other papers read at this meeting follow below. The papers of Ayers (first paper), Cramlet, Franklin, Garver, Hollcroft, Rosebrugh, Silverman and Tamarkin, Stone, Thomas, G. T. Whyburn, W. M. Whyburn, Widder, Wiener (first three papers), and Wilder were read by title. Mr. Gourin was introduced by Professor Ritt, and Professor Rosebrugh by the Secretary. 
1. Professor H. J. Ettlinger: On sequences of horizontal (step) functions.

If $E$ is a subset of points of a cube $S$ in $m$ dimensions, and $S$ is subdivided into subcells by means of $m$ sets of $n$ hyperplanes parallel to the fundamental coordinate planes, then a horizontal function of index $k=n^{m}$ is a function defined on $E$ which is constant at all points of the subset $E$ contained in a given subcell of $S$. An infinite sequence of horizontal functions is a set of horizontal functions defined for $n=1,2,3, \cdots$. If a function defined on $E$ is the limit almost everywhere of an infinite sequence of horizontal functions, it is called a function of class $M$. It is the object of the present paper to make a systematic study of the properties of functions of class $M$.

\section{Dr. T. H. Gronwall: Longitudinal vibrations of a} liquid contained in a tube with elastic walls.

An experimental method for determining the velocity of sound in a liquid consists in closing the lower end of a vertical metal tube by a membrane which is excited by an alternating current of known frequency, and filling the tube with liquid up to the point where resonance is obtained. The characteristic equation determining the frequencies of the vibrations of the liquid is set up, in the form of an infinite determinant, the elements of which contain products of Bessel functions, and, from this, approximate formulas of simple form are derived, which are directly applicable to the experimental data.

3. Dr. T. H. Gionwall: On the existence and properties of the solutions of a certain differential equation of the second order.

Under certain very general assumptions on $f(x, y)$ and $g(x, y)$, it is shown that the differential equation $(d / d x)[g(x, y) d y / d x]=f(x, y)$ with any one of the four boundary conditions (A) $y\left(x_{0}\right)=y_{1}, y\left(x_{1}\right)=y_{1}$; (B) $g\left(x_{0}, y\left(x_{0}\right)\right) y^{\prime}\left(x_{0}\right)=$ const., $y\left(x_{1}\right)=y_{1}$; (C) $y\left(x_{1}\right)=y_{1}, y(x) \rightarrow 0$ as $x \rightarrow \infty$; (D) $g\left(x_{0}, y\left(x_{0}\right)\right) y^{\prime}\left(x_{0}\right)=$ const., $y(x) \rightarrow 0$ as $x \rightarrow \infty$, has a solution which is unique. It is shown that any solution has at most one extreme, and a very general comparison theorem for the solutions of two equations of this type is established. A special case of the above equation with boundary conditions (D) occurs in the Debye-Hückel theory of solutions of strong electrolytes.

4. Dr. T. H. Gronwall: On the converse of Euler's theorem for homogeneous functions.

A homogeneous function $\phi\left(x_{1}, x_{2}, \cdots, x_{n}\right)$ of degree $k$ satisfies Euler's partial differential equation (where $m$ is any integer)

$$
\sum x_{\nu_{1}} x_{\nu_{2}} \cdots x_{\nu_{m}} \phi_{\nu_{1}}, \nu_{2}, \ldots, \nu_{m}=k(k-1) \cdots(k-m+1) \phi,
$$

where the subscripts on $\phi$ denote partial derivatives, and the summation 
extends over all subscripts of sum $m$. It is shown that the general solution of this equation is

$$
\phi=\sum \phi^{(\rho)}\left(x_{1}, \cdots, x_{m}\right)+\log x_{1} \cdot \sum^{\prime} \psi^{(\rho)}\left(x_{1}, \cdots, x_{m}\right),
$$

where $\phi^{(\rho)}$ and $\psi^{(\rho)}$ are arbitrary homogeneous functions of degree $\rho$, and the first sum extends over all the roots $\rho$ of $\rho(\rho-1) \cdots(\rho-m+1)$ $=k(k-1) \cdots(k-m+1)$, while the second sum extends the over all double roots (if any; roots of higher multiplicity do not occur).

5. Dr. Jesse Douglas (National Research Fellow): Reduction to integral equations of the problem of Platean for the case of two contours.

Let $x_{i}={ }^{1} f_{i}(t), x_{i}={ }^{2} f_{i}(t), i=1,2, \cdots, n$, represent the given two contours $\Gamma_{1}, \Gamma_{2}$, supposed contained in a euclidean space of $n$ dimensions. The $2 n$ functions $f$ are presumed to be periodic of common least positive period $\Omega$. The problem of Plateau is formulated so as to require a doublyconnected portion $M$ of a minimal surface bounded by $\Gamma_{1}, \Gamma_{2}$, and at the same time a conformal map of $M$ on a circular ring, outer radius 1 , inner radius, originally unknown, $q$. When $\Gamma_{1}, \Gamma_{2}$ are the boundaries of a doubly-connected portion of a plane, this reduces to the Riemann mapping problem. This paper reduces the Plateau problem to a system of two integral equations, for two functions $\phi_{1}(t), \phi_{2}(t)$ of the points of $\Gamma_{1}, \Gamma_{2}$ respectively, together with a third equation for the determination of $p=\log (1 / q)$.

6. Dr. Jesse Douglas: Extremals and transversality of the general calculus of variations problem of the first order in space.

Every calculus of variations problem,

$$
\text { (1) } \int F\left(x, y, z, y^{\prime}, z^{\prime}\right) d x=\text { minimum, }
$$

defines two geometric objects: first, its extremals, a family $F$ of $\infty^{4}$ curves in space; second, its transversality $T$, which is essentially a certain correspondence between lineal elements $\lambda$ and surface elements $\sigma$ such that corresponding elements have the same base point. The present paper establishes a simple criterion for determining of a given family $F$ of $\infty^{4}$ curves, together with a given transversality $T$, whether or not they can be identified respectively with the extremals and transversality of a problem of the form (1). The criterion is that the $\infty^{2}$ curves of $F$ which meet any one surface (union) $\Sigma$ transversality must always admit $\infty^{1}$ transversal surfaces. The proof is rather long, and will be published in the April, 1927, number of the Transactions of this Society.

\section{Mr. Eli Gourin: On the essentiality of arbitrary functions.}

Consider an analytic function $F\left(p, q, p^{\prime}, q^{\prime}\right)$, where $p, q$ are arbitrary functions of $x$ and $p^{\prime}, q^{\prime}$ their respective derivatives. The paper gives a set of conditions necessary and sufficient for the existence of a relation $F\left(p, q, p^{\prime}, q^{\prime}\right)=S\left(\alpha, \alpha^{\prime}\right)$, where $\alpha$ is a function of $p$ and $q$ and $\alpha^{\prime}$ is $d \alpha / d x$. 
8. Mr. H. W. Raudenbush: Note on Hilbert's thirteenth problem.

It is proved that $f$ defined by the equation

$$
f^{7}+x f^{3}+y f^{2}+z f+1=0
$$

cannot have any one of the following forms: $F(\alpha(x, y), \beta(y, z)), F(\alpha(x, y)$, $\beta(x, z))$, or $F(\alpha(x, z), \beta(y, z))$, where $\alpha, \beta$, and $F(\alpha, \beta)$ are analytic functions.

9. Professor O. E. Glenn: A theory of integers in relation to the iteration of algebraic functions.

The content and methods of this paper are presented in the form of original algorithms on the number system. The object is to develop a theory of indices in which $\Delta$ of the fundamental relation $\Delta^{r} \equiv \Delta(\bmod n)$ instead of being an integer, is a functional operation upon an integer, and $\Delta^{r}$ is its $r$ th iteration. The index $r$ is determined with considerable generality as the serial number of a term in a sequence of resultants. The resultants are modular and appertain to the theory due to Stieltjes. Applications are noted, to problems relating to two-dimensional residue systems.

10. Mr. T. W. Moore: On the invariant combinants of two binary quintics. Preliminary report.

In a study of the rational space quintic curve the author has been led to the set of combinant forms of two binary quintics. From work of Gordan and Stroh it is known that the complete system of combinants of the two quintics is the same as the simultaneous system of covariant forms of the binary octavic and quartic, which are the first and third transvectants respectively of the two given forms. In turn, this system is identical, except for invariant factors, with that of another binary octavic apolar to the two quintics, and so the number of combinant forms is known. Starting with the three syzygies given by L. Berzolari in vol. 7 of the Rendiconti di Palermo, this paper sets up the complete system of invariant combinants of the two forms of the fifth order, together with the necessary covariant forms required for this purpose.

\section{Dr. Louis Weisner: Invariant theory of plane n-points} and n-lines.

The author investigates the projective invariant theory of complete $n$-points and $n$-lines. The work naturally falls into two parts: the consideration of concomitants expressed in terms of the coordinates of the vertices of the $n$-points and of the sides of the $n$-lines, and the consideration of concomitants expressed in terms of the coefficients of the degenerate ternary line-curves and point-curves defined by the $n$-points and $n$-lines respectively. Finiteness theorems are proved, a symbolic theory is developed, and a theorem somewhat analogous to Hermite's reciprocity law is proved. 
12. Professor James Pierpont: Optics in spaces of constant curvature. Second paper.

In a paper read at the last Annual Meeting of the Society, the author showed that in space of constant non-vanishing curvature, central optical collineation gives only an image congruent with the object. Thus this elegant method is not available in elliptic or hyperbolic spaces. However, instead of rays of light one may use waves of light, adopting the methods laid down by C. S. Hastings (On certain new methods and results in optics, Memoirs of the National Academy vol. 6 (1893), p. 37). In general it is found that the results are similar to those of euclidean optics. As an example, the familiar formula for a convex lens, $(1 / r)+\left(1 / r_{1}\right)=((1-n) / n)\left((1 / l)+\left(1 / l_{1}\right)\right), n<1$, becomes for hyperbolic spaces, $\operatorname{ctnh}\left(\rho_{1} / R\right)+\operatorname{ctnh}(\rho / R)=((1-n) / n)\left(\operatorname{ctnh}(\lambda / R)+\operatorname{ctnh}\left(\lambda_{1} / R\right)\right)$, and a similar formula for elliptic space.

13. Dr. M. H. Stone: A characteristic property of certain sets of trigonometric functions.

In this paper the nature of a closed normal orthogonal set of functions whose derivatives constitute an orthogonal set is discussed. It is shown that when certain auxiliary conditions are satisfied by the functions of the set they are necessarily trigonometric and satisfy a differential system of the second order.

14. Dr. M. H. Stone: The normal frequency function and general frequency functions.

The problem considered is that of approximating to an arbitrary function by a linear combination of functions of the form $e^{-\lambda(x-\mu)^{2}}$, where $\lambda$ and $\mu$ are constants, $\lambda>0$. Three theorems concerning the possibility of such representation are obtained with the aid of the theorem of Riesz-Fischer.

15. Professor Norbert Wiener: On the closure of sets of trigonometric functions.

The author discusses the closure of the set of functions $\cos \lambda_{n} x$.

16. Professor Norbert Wiener: A new definition of almost periodic functions.

The author gives a new necessary and sufficient condition for a function to be almost periodic.

17. Professor Norbert Wiener: Laplacians and linear functionals.

The author develops a new theory of functions of limited total variation in $n$ dimensions, connected with the Laplacian operator. $\mathrm{He}$ applies this to the theory of linear functionals and to that of multidimensional trigonometric developments. 
18. Mr. G. T. Whyburn: Concerning the complementary domains of continua.

The author shows that (1) if the complementary domains of a boundel continuum $M$ have the property that for any number $\epsilon>0$ at most a finite number of them are of diameter $>\epsilon$, and $P$ is any point of $M$ not belonging to the boundary of any complementary domain of $M$, then (a) for every number $d>0, M$ contains a subcontinuum of diameter $<d$ which separates $P$ from all those points of the plane whose distance from $P$ is $>d$, and (b) $M$ is regular at $P$; (2) the complementary domains of a bounded continuum $M$ have the above mentioned property if and only if for every point $P$ of $M$ and every number $d>0$ there exists a continuum $N$ of diameter $<d$ which separates $P$ from all those points of the plane whose distances from $P$ are $>d$ and which is a subset of $M+$ a finite number of the complementary domains of $M$; and (3) if the complementary domains of an indecomposable continuum $M$ have the above mentioned property, then $M$ is the boundary of at least one of its complementary domains.

19. Mr. G. T. Whyburn: Concerning the sum of the boundaries of the complementary domains of a plane continuous curve.

In this paper it is shown that if $H$ denotes the point set obtained by adding together the boundaries of all the complementary domains of a continuous curve, $M$ every subcontinuum of which is a continuous curve, then (1), the set $M-H$ contains no continuum, and (2), the set $H$ is connected. It is further shown that if $M$ is any continuous curve which contains no domain, then in order that the point set $H$ obtained by adding together the boundaries of all the complementary domains of $M$ should be connected, it is necessary and sufficient that the set $M-H$ should contain no closed subset which separates the plane.

20. Mr. G. T. Whyburn: Concerning the open subsets of a continuous curve.

A boundary point $P$ of a domain $D$ in a space $E$ will be said to be regularly accessible from $D$ provided it is true that for every positive number $\epsilon$, a $\delta_{\epsilon}>0$ exists, such that every point $X$ of $D$, whose distance from $P$ is $<\delta_{\epsilon}$, may be joined to $P$ by an arc $X P$ of diameter $<\epsilon$, and such that $X P-P$ lies in $D$. In this paper it is shown that (1) if $E$ is the euclidean plane, the above definition is equivalent to accessibility from all sides as introduced by Schoenflies; (2) if $E$ is any plane continuous curve, every boundary point of a domain $D$ is regularly accessible from $D$ if and only if $D$ has property $S$ (R. L. Moore, Fundamenta Mathematicae, vol. 3, p. 232); (3) if $E$ is a plane continuous curve, every subcontinuum of which is a continuous curve, every boundary point of any domain $D$ is regularly accessible from $D ;(4)$ a connected open subset of a plane continuous curve is cyclically connected if and only if it has no cut point; (5) if $M$ and $N$ are continuous curves, $N \subset M$, then every maximal connected subset of $M-N$ has property $S$. 
21. Professor W. M. Whyburn: Existence and oscillation theorems for second-order differential systems whose boundary conditions contain definite integrals.

In this paper, the pair of differential equations $d y / d x=K(x, \lambda) z$, $d z / d x=G(x, \lambda) y$, together with boundary conditions, one of which contains a definite integral (Lebesgue), is treated. The functions $K$ and $G$ are assumed continuous in $\lambda$ for each $x$ on the interval of definition, and summable in $x$ for each $\lambda$ on the $\lambda$ interval. For every $x$ and every $\lambda$ on the intervals of definition, $K$ and $G$ are less than (or equal to) a summable function of $x$. In the presence of the restrictions that $K / G$ and $A / G$ be non-decreasing functions of $x$ for each fixed $\lambda$, where $A(x, \lambda)$ is the coefficient of $y(x, \lambda)$ in the integral condition, the existence of an infinite set of characteristic values of $\lambda$ is established. Oscillation theorems are given for the solutions of the system, for linear combinations of these solutions, and for indefinite integrals containing these solutions in their integrands. Similar theorems are proved for $z(x, \lambda)$.

22. Professor W. M. Whyburn and Mr. G. T. Whyburn: On the Porter-Vitali theorem.

The authors give a new and shorter proof of a theorem due to Arzela that every bounded sequence of functions which are analytic in a closed region $R$ contains a subsequence which converges to an analytic limit function everywhere in $R$. On the basis of this theorem they obtain a very short proof of the Porter-Vitali theorem that if a uniformly bounded sequence of functions analytic in a region $R$ converges over a set of points having a limit point in $R$, then the whole sequence converges throughout $R$ to an analytic limit function. A new application of these theorems is given.

23. Professor R. L. Wilder: A point set which has no true quasi-components, and which becomes connected upon the addition of a single point.

Sierpinski has given (Fundamenta Mathematicae, vol. 2 (1921), pp. 81-85) an example of a point set which has no true quasi-components, but which, on the addition of a single point, contains a quasi-component. In the present paper the author shows, by an example, that there exists a point set $M$ which has no true quasi-component, and which, on the addition of a single point, becomes connected. Incidentally, the set $M$ furnishes another example of a certain type of point set recently constructed by Mazurkiewicz (Fundamenta Mathematicae, vol. 2, pp. 201-205); i. e., a quasi-connected set $M$ of which every two points are separated in $M$.

24. Mr. W. L. Ayres: Note on a theorem concerning continuous curves.

In a number of recent applications the following theorem has been found useful: If $M$ is a plane continuous curve (bounded or unbounded) 
and $N$ is a bounded continuous curve which is a subset of $M$, then, for any positive number $\epsilon, M-N$ contains at most a finite number of maximal connected subsets of diameter greater than $\epsilon$. This is an extension of a result previously presented (Concerning the arcs and domains of a continuous curve, this Bulletin, vol. 32 (1926), p. 37).

25. Professor T. R. Hollcroft: On higher point correspondences.

When both planes are multiple, only $(2,2)$ and $(2,3)$ point correspondences have been classified and studied in detail. In this paper, $(3,3)$ point correspondences between two planes are completely classified. Fifteen types of general $(3,3)$ point correspondences and fifteen types of $(3,3)$ compound involutions are found, and the characteristic features of each type are given. The number of types each of $(2,4)$, $(3,4)$, and $(4,4)$ point correspondences and the general characteristics of these correspondences are also found. As in the case of $(1,4)$ involutions when multiplicity of one or both planes is greater than four, no new features are introduced.

26. Dr. Raymond Garver: A theorem concerning Tschirnhaus transformations.

The following theorem is proved: Every Tschirnhaus transformation of degree $n-1$ which transforms a sufficiently general principal equation (in $x$ ) of degree $n>4$ into another principal equation can be expressed as a linear function of $\phi$ and $\psi$, where $\phi$ and $\psi$ are polynomials (in $x$ ) with special properties. (By a principal equation we mean one in which the second and third coefficients are zero.) Transformations of this kind are especially convenient to work with, and we thus show that in certain problems we suffer no loss of generality by restricting our attention to them.

27. Dr. Raymond Garver: Division algebras of order sixteen.

In this paper a type of division algebra of order sixteen, based on a root of a quartic equation irreducible in the field of rational numbers and having the Galios group (1), (12) (34), (13) (24), (14) (23), is set up, in accordance with the conditions given in Dickson's New division algebras (Transactions of this Society, April, 1926). For this case there are six conditions, which restrict the coefficients of the quartic and the constants of multiplication of the algebra. Cases are exhibited where all the conditions are satisfied.

28. Mr. C. M. Cramlet: The derivation of projective algebraic invariants by tensor algebra.

In this paper it is proved that any algebraic invariant can be readily written as an inner product of the fundamental tensors, which are the coefficients of the forms, and the determinant tensor

$$
\delta_{s_{1} \ldots s_{n}}^{r_{1} \ldots r_{n}}
$$


The methods apply also to non-symmetric tensors. Although invariants written in this manner are explicitly expressed, the transition to the classical symbolic form involves a mere change of notation.

29. Dr. D. V. Widder (National Research Fellow): The transformations of the special relativity theory.

In the special relativity theory it is desired to determine those real one-to-one transformations $x_{i}=x_{i}\left(y_{1}, y_{2}, y_{3}, y_{4}\right)$ which make

$$
d x_{1}^{2}+d x_{2}^{2}+d x_{3}^{2}-d x_{4}^{2}=\mu\left(d y_{1}^{2}+d y_{2}^{2}+d y_{3}^{2}-d y_{4}^{2}\right),
$$

where $\mu$ is a function of $y_{1}, y_{2}, y_{3}, y_{4}$, in general different from zero. In this paper, all possible values of $\mu$ are determined, and the corresponding transformations are set up. It is found that the case $\mu=$ constant is the only one that leads to a transformation continuous throughout space. This continuity is a requirement of the relativity theory, so that in this way all other values of $\mu$ may be excluded.

30. Dr. J. M. Thomas (National Research Fellow): On systems of total differential equations.

It is shown that, in applying the theorem given by $O$. Veblen and the writer (Annals of Mathematics, (2), vol. 27 (1926), p. 290) to systems of tensor differential equations, the process of differentiation can be chosen as partial or covariant at will. By adopting the latter alternative, the theorems for the existence of homogeneous first integrals in the geometry of paths (O. Veblen and T. Y. Thomas, Transactions of this Society, vol. 25 (1923), pp. 551-608) are readily deduced as special cases.

31. Professor J. W. Young: On the definition of the ScorzaDickson algebras.

The definition of "an algebra $A$ over a field $F$ " given by Dickson in his Algebras and their Arithmetics, pp. 9, 10, consists of five groups of postulates I-V, of which groups II and III refer exclusively to multiplication between the elements of $A$ and the marks of $F$. In the present paper it is shown that for a large part at least, if not for all, of the later theory the postulate groups II and III are unnecessary and all reference to a field $F$ can be avoided. A slight modification of postulate group I (insuring merely that $A$ forms an abelian group with respect to addition) and postulate groups IV and V constitute a sufficient basis from which to derive all the theorems in the first three chapters of Dickson's book. This is accomplished by using the idea of the partitions of the addition group (see abstract of a paper by the author presented to the Society September 9, 1926, this Bulletin, vol. 32, p. 588).

32. Professor Norbert Wiener and Dr. Robert Schmidt: A general form of Tauberian theorem.

By using the Fourier integral and the related theory of complex moments, the authors obtain a very general Tauberian theorem, in- 
cluding most known Tauberian theorems as special cases, in particular, the Tauberian theorem for Lambert series, from which the prime number theorem may be deduced, as Hardy has shown.

33. Professor Norbert Wiener: The approximation theorem for almost periodic functions.

The author gives a new proof of the approximation theorem for almost periodic functions.

34. Mr. W. L. Ayres: On the separation of points of a continuous curve by arcs and simple closed curves.

If $x$ and $y$ are distinct points of a plane continuous curve $M$ (bounded or unbounded) and $M_{x y}$ denotes the set of all points $P$, such that $P$ lies on some simple continuous arc of $M$ whose end points are $x$ and $y$, then $M_{x y}$ is a continuous curve. With the use of this result, the following two theorems are proved: (1) a necessary and sufficient condition that $M$ contain a point which separates $x$ and $y$ in $M$ is that every two simple continuous arcs of $M$ whose end points are $x$ and $y$ have a point distinct from $x$ and $y$ in common; (2) if $M$ is bounded, a necessary and sufficient condition that $M$ contain a simple continuous arc which separates $x$ and $y$ in $M$ is that $M-(x+y)$ contain only one maximal connected subset which has both $x$ and $y$ as limit points; (3) if $M$ is bounded, a necessary and sufficient condition that $M$ contain a simple closed curve $J$, such that one of the two points $x$ or $y$ is interior to $J$ and the other is exterior, is that both $x$ and $y$ do not lie on the boundary of the same complementary domain of $M$. In (2), a point is considered to be a special case of an arc.

\section{Mr. W. L. Ayres: Concerning the boundaries of domains of a continuous curve.}

If $D$ is a domain of a plane continuous curve $M, B$ is its $M$-boundary, and $P$ is a point of $M-(D+B)$, then the $M$-boundary of the maximal connected subset of $M-(D+B)$ containing $P$ is called the $M$-boundary of $D$ with respect to $P$. These theorems are then proved: if $N$ is a continuous curve which is a subset of $M$, every closed and connected subset of the $M$-boundary of a complementary $M$-domain of $M$ is a continuous curve; (2) if $\beta$ is the $M$-boundary of $D$ with respect to $P$, then $\beta$ is the entire $M$-boundary of some $M$-domain which contains $D$; (3) if every maximal connected subset of $B$ is a continuous curve and either $D$ or the maximal connected subset of $M-(D+B)$ which contains $P$ is bounded, then every maximal connected subset of the $M$-boundary of $D$ with respect to $P$ is either a point, an arc, or a simple closed curve, and if one maximal connected subset is a simple closed curve $J$, then $J$ is the entire $M$-boundary of $D$ with respect to $P ;(4)$ if $\beta$ is the $M$-boundary of $D$ with respect to $P, R$ is the maximal connected subset of $M-(D+B)$ containing the point $P$, and $Q$ is any point of the maximal connected subset of $M-(R+\beta)$ which contains $D$, then $\beta$ is the $M$-boudnary of $R$ with respect to $Q$. 


\section{Professor Edward Kasner: Equilong geometry.}

In previous papers, the author has studied various questions of equilong geometry mainly with the object of comparison with the analogous (but in general more difficult) questions of conformal geometry (see Proceedings of the Fifth International Congress, Cambridge, 1912, vol. 2, pp. 81-87, and this Bulletin, vol. 23 (1917), pp. 341-347). In the present paper, he discusses more fully the analogy of isothermal systems of curves in equilong geometry, equilong symmetry (see this Bulletin, vol. 24 (1918), p. 471), and the invariants of irregular analytic curves. Some types have invariants and some have not, in general as in the conformal theory (see Transactions of this Society, vol. 16 (1915), p. 339), but the detailed results are different.

37. Professor Edward Kasner: Irregular differential invariants. I: General theory.

The ordinary concept of differential element and differential invariant relates to curves as represented by polynomials or power series with integer exponents. The author has extended this theory by allowing irregular elements or curves where the series involve fractional exponents. This theory has been carried out for the conformal group of the plane in his paper in the Transactions of this Society, vol. 16 (1915), pp. 333-349 (see also this Bulletin, vol. 21 (1915), p. 280), and for the equilong group in his first paper read at this meeting of the Society. In the present paper he considers continuous groups in general, and in later papers will discuss the irregular invariants of the infinite groups of contact transformations and of point transformations, as well as the finite projective and affine groups.

38. Professor Philip Franklin: Analytic functions with assigned values at an infinite number of points.

The question of determining when the values of a function at an infinite set of points in a finite region determine a function analytic in this region, and if so the function in question, has recently been raised by T. H. Hildebrandt (this Bulletin, vol. 32 (1926), p. 552). This question is answered in the present note.

39. Professors L. L. Silverman and J. D. Tamarkin: On the generalization of Abel's theorem for certain definitions of summability.

Abel's classical theorem on power series was first generalized by Frobenius, for the case when the series is summable by arithmetic means. Subsequently, Abel's theorem has been extended by various authors to other definitions of summability: Cesàro's and Hölder's of all orders, Borel's and Euler's. The purpose of the present paper is to discuss the extension of Abel's theorem to the general class of analytically regular transformations (Hurwitz and Silverman, Transactions of this Society, 
vol. 18 (1917), pp. 1-20); the result obtained is that among these transformations only those equivalent to Hölder's admit of the generalization of Abel's theorem. The general regular transformations permutable with $M$, as given by Hausdorff, are also discussed, and, finally, Abel's theorem is extended to the definition of summability of Nörlund.

\section{Professor T. R. Rosebrugh: Quantic determinants.}

In this discussion, any number of linear substitutions are given, independent of each other as to coefficients and variables. The substitution is formed which gives all corresponding new monomials linearly in terms of all the possible old monomials whose factors are taken from all substitutions, and from each to a total degree assigned to that substitution. The algebraic composition of this determinant is found without assumptions by examination of the effect of certain linear partial-derivative operators upon it. From this general result, by specialization in different directions, theorems previously given by Kronecker, Faà di Bruno, and Scholtz may be obtained.

\section{R. G. D. RichaRDSON,}

Secretary

\section{A CORRECTION}

In the last line of page 140 of the March-April issue (this Bulletin, vol. 33 (1927)),

instead of $\quad\left\{f(x)-\left[T_{n}(x)\right]\right\}^{2}, \quad$ read $\quad\left\{f(x)-\left[T_{n}(x)\right]^{2}\right\}^{2}$. 